PHYSICAL REVIEW FLUIDS 1, 012501(R) (2016)

\title{
Pressure evolution in the shear layer of forming vortex rings
}

\author{
Kristy Schlueter-Kuck* \\ Department of Mechanical Engineering, Stanford University, Stanford, California 94305, USA \\ John O. Dabiri ${ }^{\dagger}$ \\ Department of Civil and Environmental Engineering, and Department of Mechanical Engineering, \\ Stanford University, Stanford, California 94305, USA \\ (Received 10 February 2016; published 2 May 2016)
}

\begin{abstract}
This study investigated the relationship between the pinch-off of axisymmetric vortex rings and the evolution of pressure in the shear layer being entrained into the vortex rings. A piston-cylinder apparatus was used to generate the vortex rings, and five cases of constant piston acceleration over distances ranging from zero (impulsive start) to eight piston diameters were investigated. It was determined that increasing the distance over which the piston accelerated increased the dimensionless formation time at which the vortex ring pinches off, consistent with previous observations. A limiting value of vortex ring formation number of approximately seven is approached when the piston is accelerated over more than six piston diameters. For each case, the evolution of pressure in the shear layer was calculated based on PIV measurements of the velocity field and spatial integration of the corresponding pressure gradients using a recently developed algorithm. Plots of the shear layer pressure in X-T diagrams aided in identifying key features of the pressure associated with the evolution of vortex rings, including a high-pressure region that forms behind the leading ring. By extrapolating the motion of this high-pressure region back to the nozzle exit plane in the X-T diagram, its time of first appearance can be estimated. It is found that the appearance of the extrapolated local pressure maximum in the shear layer at the nozzle exit plane coincides with vortex ring pinch-off, as conventionally quantified by the vortex ring formation number.
\end{abstract}

DOI: 10.1103/PhysRevFluids.1.012501

\section{INTRODUCTION}

The study of vortex shedding of interest to fluid mechanicians in part due to the potential for predicting and controlling when and how vortex shedding will occur. As a step toward understanding these processes in general, a specific manifestation of this problem, the study of pinch-off of axisymmetric vortex rings, can provide useful insights. The pinch-off of vortex rings has been well characterized and thoroughly studied for several decades [1-4]. Gharib et al. [5] characterized the vortex ring pinch-off process for vortex rings generated in a laboratory setting by pushing a piston through a hollow cylinder. They defined the vortex formation time, or time normalized by the piston diameter $D$ and the running average of the piston velocity $\overline{U_{p}}$, as $t^{*}=\overline{U_{p}} t / D$. The study found that for vortex rings generated with a high stroke ratio piston motion, vortex rings pinched off at formation times between 3.6 and 4.5. The nondimensional time at which pinch-off occurred was termed the formation number.

While the somewhat universal nature of formation number is intriguing, and useful for predicting pinch-off in axisymmetric starting flows, it has some limitations. The first is that it is possible to increase or decrease the formation number beyond the typical range by manipulating certain experimental parameters. For example, Dabiri and Gharib [6] were able to increase the formation

\footnotetext{
*kristy@stanford.edu

†jodabiri@stanford.edu
} 


\section{KRISTY SCHLUETER-KUCK AND JOHN O. DABIRI}

number to 8.0 by temporally varying the nozzle exit diameter, confirming numerical predictions by Mohseni et al. [7]. Conversely Krueger et al. [8] reduced the formation number substantially by introducing a background coflow during vortex ring formation.

Several explanations for the physical mechanism underlying pinch-off have been proposed that are consistent with the observed variations in formation number. Gharib et al. [5] proposed that vortex rings pinch off when vortex generator is no longer able to supply the energy needed to ensure that a vortex ring propagating at a constant velocity has maximal energy with respect to circulation and impulse preserving perturbations. Mohseni et al. [7] and Shusser and Gharib [9] proposed that pinch-off is initiated when the maximum axial velocity in the vortex ring exceeds the maximum velocity in the shear layer of the trailing jet. Still others have proposed that pinch-off is the result of a developing shear layer instability in the trailing jet [10].

The multitude of potential explanations of pinch-off, combined with the demonstrated possibility of manipulating the formation number, make predicting pinch-off difficult. One recent method for addressing these issues was introduced by Lawson and Dawson [11], who proposed a pressure-based mechanism for vortex ring pinch-off. The authors indirectly observed a local pressure maximum, termed the trailing pressure maximum (TPM), on the axis of symmetry for turbulent vortex rings produced using a synthetic jet. The appearance of the TPM was coincident with vortex ring pinch-off. The use of a pressure-based indicator of pinch-off is appealing because its characterization can be independent of the geometry of the apparatus used to produce the vortex rings.

We hypothesized that the pressure evolution in the shear layer feeding the developing vortex ring would provide a more precise signature associated with pinch-off than the centerline flow studied by Lawson and Dawson [11]. Examination of the evolution of pressure in the shear layer facilitates a more timely determination of pinch-off than current methods, which require measurement of the circulation in the vortex ring well past the actual formation number [5]. In highly unsteady flows exhibiting rapid vortex ring breakdown, such measurements are not possible, leaving the pressure-based approach as potentially the only alternative.

The following section describes in detail the velocity profiles tested and the experimental apparatus used. The methods used for data analysis are also discussed. Section III highlights the results from the conventional circulation analysis to determine the formation number for each velocity profile. In Sec. IV the features observed in an X-T diagram of the pressure in the shear layer are described, and the method for estimating pinch-off from these plots is discussed.

\section{METHODS}

Laminar vortex rings were generated by accelerating fluid through a hollow cylinder with inner diameter $D$ of $2.49 \mathrm{~cm}$ mounted in the bottom of a water tank with cross-sectional area of $61 \mathrm{~cm}$ by $61 \mathrm{~cm}$ and height of $91 \mathrm{~cm}$. A piston was moved through the cylinder under the action of a $8.3 \mathrm{~cm}$ diameter syringe actuated using a stepper motor (Omega OMHT34-485) and driver (STR8). The stepper motor was controlled using an Arduino code. The rotational motion of the stepper motor was converted into linear motion using a system of gears and a lead screw to move the plunger in the syringe (Fig. 1).

Several temporally varying velocity profiles were investigated. All prescribed velocity programs had a maximum velocity of $7.3 \mathrm{~cm} / \mathrm{s}$ and were accelerated linearly (with respect to the formation time) from rest to the maximum velocity while the piston moved over a distance of $L / D=t_{a}^{*}=0$, $2,4,6$, and 8 . After the maximum velocity was reached, the piston traveled at a constant speed $u_{\max }$ until reaching a maximum stroke ratio, $L / D_{\max }$, of 8 , at which time the piston was decelerated impulsively to rest. The actual velocity profiles were estimated by using an integration of the velocity flux through a control volume surrounding the nozzle exit, where the velocity was derived from the particle image velocimetry (PIV) analysis. Inherent in the piston velocity estimation is the assumption of axisymmetry of the flow, which is valid near the nozzle exit except at very small piston velocities. Both the prescribed and the actual velocity profiles are shown in Fig. 2. Three runs were performed for each velocity profile. 
(a)

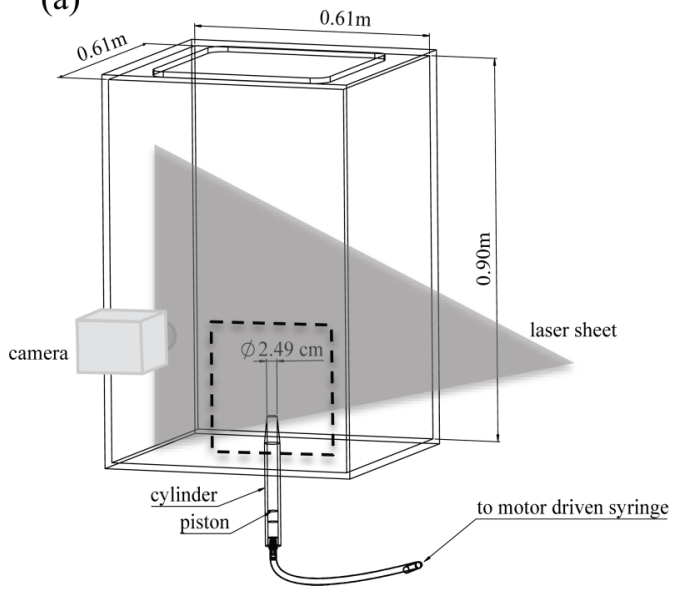

(b)

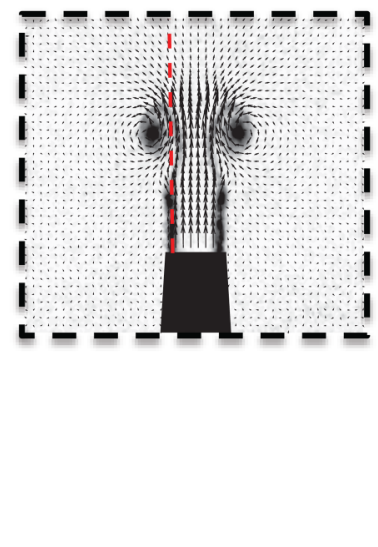

FIG. 1. Experimental setup. (a) a schematic of the tank used to generate vortex-rings using a piston-cylinder assembly mounted in the bottom of the tank. (b) A subsection of a sample velocity field derived using PIV analysis. The red dotted line coincides with the center of the shear layer.

Particle image velocimetry was performed to obtain measurements of the velocity field. A continuous, $1 \mathrm{~W}$, 532-nm DPSS laser system (Laserglow Technologies) was used in conjunction with a plano-concave cylindrical lens (Thorlabs) with a $-3.9 \mathrm{~mm}$ focal length to create a laser sheet aligned with the nozzle cross section. The flow was seeded with $13 \mu \mathrm{m}$ silver-coated hollow glass spheres (Potters Industries, LLC). The flow was imaged using a high-speed camera with 1088 by 2048 pixel resolution (Emergent Vision Technologies) at a rate of 100 frames per second.

Raw images were processed using DaVis image software (Lavision Technologies) to obtain two-dimensional PIV measurements. A double-pass PIV algorithm was used with decreasing window size. The first pass used 32 pixel by 32 pixel windows with $50 \%$ overlap, and the second pass used 16 pixel by 16 pixel windows, again with $50 \%$ overlap. The final window size resulted in vector spacing of $1.17 \mathrm{~mm}$. Spurious vectors were removed using a median filter with universal outlier

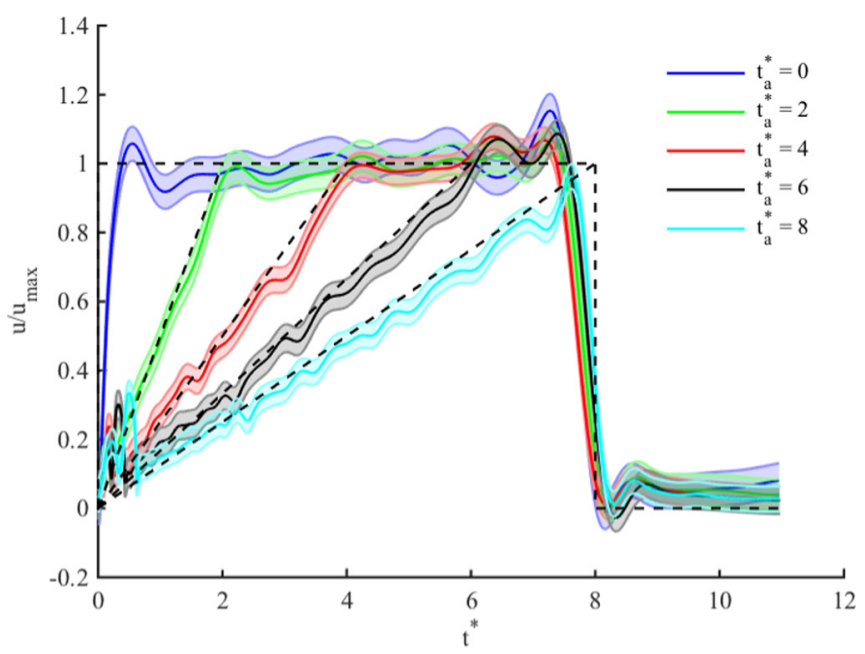

FIG. 2. Prescribed (dotted line) and actual piston velocity profiles for all acceleration distances, $t_{a}^{*}$. Shaded regions indicate measurement uncertainty. 
detection, and replaced using interpolation. Analysis of temporal velocity fluctuations at a point in the jet near the nozzle exit for the constant velocity case indicated that the error in the velocity data is less than $2 \%$. Propagation of this error through the calculation of circulation led to error in the circulation data of less than $2 \%$ as well.

In order to examine the pressure evolution in the shear layer of developing vortex rings, pressure fields were derived from the PIV velocity vector fields using a recently developed computational algorithm [12]. This method derives the pressure gradient field using the Navier-Stokes equation and integrates it along several paths throughout the domain. In contrast to an earlier method by Liu and Katz [13], median polling, rather than averaging, is then used to select the best estimate for the pressure at every point. Further details concerning this algorithm can be found in Dabiri et al. [12], which also presents a validation of the model using several test cases. A review of other pressure calculation techniques derived from PIV data can be found in van Oudheusden [14].

A particularly relevant validation performed on the present pressure algorithm, detailed in Appendix 1 of Dabiri et al. [12], studies the effect of adding Gaussian noise to the velocity field of a computational simulation of $2 \mathrm{D}$ flow past a square. The authors found that for signal-to-noise ratios comparable to those in typical PIV data sets, the quantitative pressure estimates are consistent with the pressure values found when noise is omitted. They also concluded that due to the median polling algorithm, spatial discontinuities in pressure are a robust indicator that the noise in the velocity data is unacceptably large. No such discontinuities were observed in the present study. Moreover, for spatial resolutions comparable to the present study, the error in the instantaneous pressure was less than $5 \%$, comprising a combination of model error and numerical round-off error.

\section{CIRCULATION ANALYSIS}

In order to determine the formation number of each vortex ring, i.e., the the formation time at which the vortex ring pinches off, a circulation analysis similar to the approach taken in Gharib et al. [5] was performed. Specifically, the circulation in a symmetry plane of the flow domain was measured throughout the ring development. Additionally the circulation in an azimuthal cross section of the fully developed leading vortex ring (LVR) was calculated for times when the ring had separated sufficiently from the trailing jet that its circulation could be measured independently. The
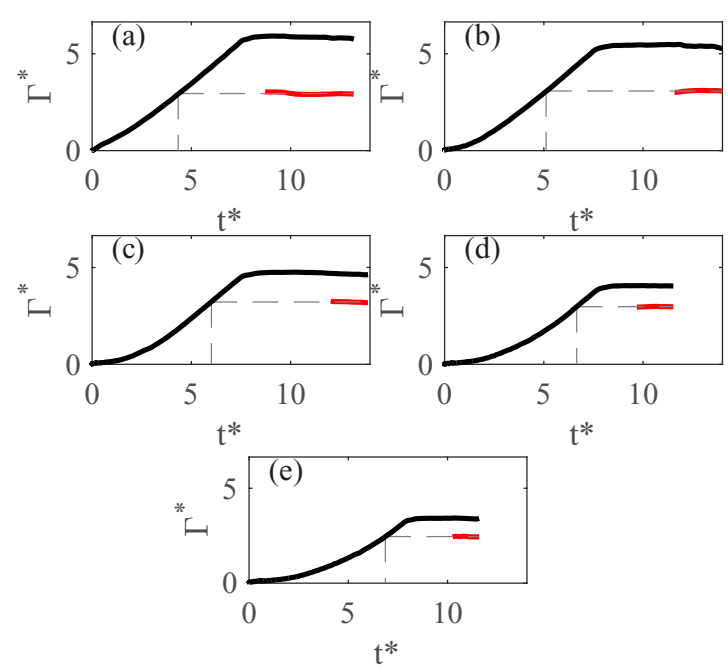

FIG. 3. Evolution of circulation for (a) $t_{a}^{*}=0$, (b) $t_{a}^{*}=2$, (c) $t_{a}^{*}=4$, (d) $t_{a}^{*}=6$, (e) $t_{a}^{*}=8$. Circulation in cross section of flow field given by black line. Circulation in leading vortex ring given by red line. Dotted lines show calculation of formation number from the average circulation in the ring. 
circulation of the vortex ring was then compared to the circulation of the total flow field to determine the formation time $t^{*}$ when the circulation in the LVR was first present in the flow domain.

The results of this circulation analysis are presented in Fig. 3, where the total circulation in a symmetry plane of the flow field and in the LVR, both nondimensionalized as $\Gamma^{*}=\Gamma / u_{\max } D$, are plotted as a function of formation time for each velocity profile examined. For the constant velocity case, i.e., $t_{a}^{*}=0$, the total circulation increased linearly until the piston stopped moving at approximately $t^{*}=8$. The resulting formation number is 4.4 , which is consistent with the results of previous studies [5]. As the acceleration distance $t_{a}^{*}$ is increased, the rate of circulation growth also increases while the piston is accelerating, and the percentage of the total circulation present in the fully developed vortex ring increases as well. This results in an increase in formation number. For $t_{a}^{*}=2$, the formation number ranges from 5.0 to 5.1; and for $t_{a}^{*}=4$, the formation number ranges from 5.9 to 6.1. As the acceleration distance is further increased, the formation number begins to plateau, with $t_{a}^{*}=6$ resulting in formation numbers ranging from 6.5 to 6.7 and formation numbers from 6.6 to 6.9 observed for $t_{a}^{*}=8$.

The observed upper limit on the formation numbers that can be achieved with the linearly accelerating piston velocity profiles is consistent with the analysis of Kaplanski and Rudi [15], which found theoretical upper limits significantly greater than 4 for formation numbers of viscous vortex rings.

\section{PRESSURE ANALYSIS}

An effective way to visualize the time history of pressure in the shear layer is to plot the time-dependent pressure along a line extending perpendicular to the exit plane of the nozzle and centered in the middle of the shear layer near the nozzle edge as it evolves in time (see Fig. 1). This yields a contour plot of pressure in the shear layer as a function of distance from the nozzle exit (i.e., horizontal axis) and time (i.e., vertical axis). A plot corresponding to the $t_{a}^{*}=0$ case is shown in Fig. 4, with the $y$ axis coincident with the nozzle exit plane. Several important flow features become evident using this method, as identified using Roman numerals on the figure. These include

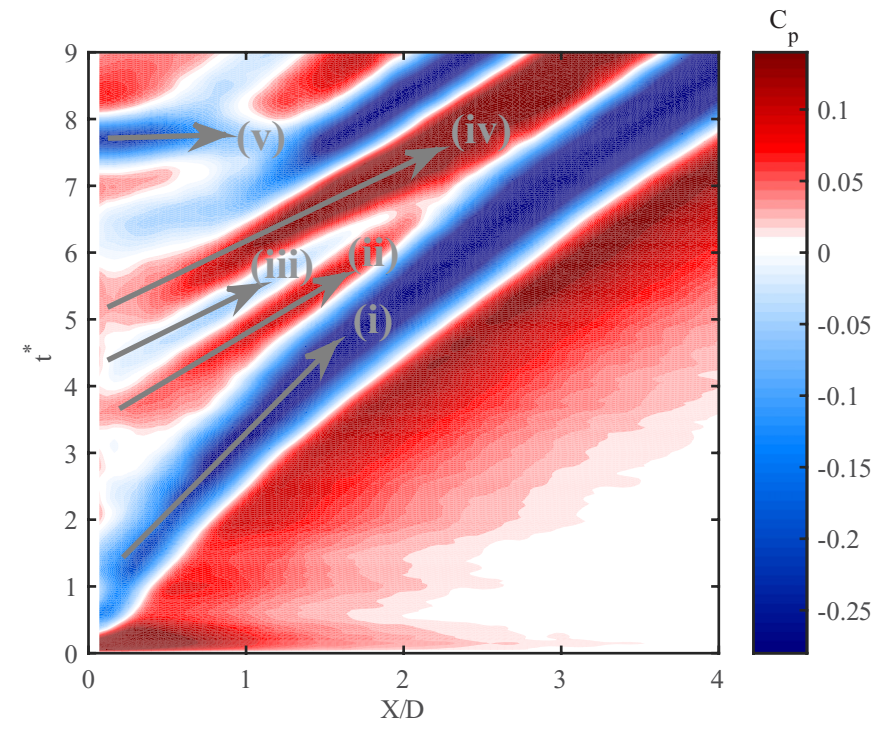

FIG. 4. Pressure evolution in the shear layer, $t_{a}^{*}=0$ (i) low-pressure region coincident with leading vortex ring core, (ii) first TPM, (iii) secondary vortex ring core, (iv) secondary TPM, (v) low-pressure wave due to piston deceleration. Pressure coefficient $C_{p}=P / \rho u_{\max }^{2}$. 


\section{KRISTY SCHLUETER-KUCK AND JOHN O. DABIRI}

the low-pressure region that coincides with the core of the vortex ring (i), which moves away from the nozzle as time progresses. Near the nozzle exit plane at approximately $t^{*}=3.5$, a high-pressure region develops behind the vortex core (ii). This is equivalent to the trailing pressure maximum (TPM) described by Lawson and Dawson [11].

Subsequently a secondary vortex ring develops behind the TPM, and has a core aligned with the low-pressure region indicated by (iii). As the secondary vortex ring develops, a secondary TPM forms behind it (iv). At approximately $X / D=2.0$ and $t^{*}=6.7$, the primary and secondary vortex rings merge, a phenomena observed by Gharib et al. [5]. This vortex merger is a result of the action of the induced velocity of the leading ring on the secondary ring. Concurrently the primary TPM (ii) decays in strength. After the two rings merge, the high-pressure region that was initially behind the secondary ring (iv) grows in magnitude and evolves into the large high-pressure region behind the fully developed leading vortex ring. After $t^{*} \approx 6.5$, the low-pressure region aligned with the leading ring core (i) and the high-pressure region behind it (iv) traverse downstream at a relatively constant rate. The final important feature highlighted by this figure is the quickly propagating low-pressure region that develops near the nozzle exit and is evident up to one diameter away from the nozzle exit plane at $t^{*} \approx 8(\mathrm{v})$. This is associated with the impulsive stopping motion of the piston at the end of its motion.

One reason that Lawson and Dawson [11] hypothesize that a local pressure maximum behind the leading vortex ring is associated with pinch-off is that a high-pressure region creates a barrier that repels fluid particles. In other words, fluid in front of the TPM tends to stay ahead of it, while fluid behind the TPM tends to stay behind it. These kinematics are consistent with the pinch-off process. In these experiments, we observe a distinct high-pressure region behind the fully formed leading vortex, and by tracing its origin back to the nozzle exit plane in the X-T diagram, we can estimate pinch-off as the nondimensional time that this TPM first appears at the nozzle exit plane. In the case described above, the TPM of interest is the second TPM, because the first TPM decays when the leading and secondary vortex rings merge.

It is important to note that the TPM as identified by Lawson and Dawson is the first pressure maximum observed behind the leading vortex ring. This XT diagram highlights the fact that in this case the first TPM is not associated with pinch-off, since fluid ejected from the nozzle between the first and the second TPM eventually becomes entrained in the leading vortex ring. Hence, the pinch-off criterion proposed by Lawson and Dawson regarding the appearance of a TPM behind the leading vortex ring is necessary, but not a sufficient condition. Sufficiency depends on the TPM being robust to vortex merger. While the origin of the TPM in Fig. 4 is fairly clear, this is not always the case. When the vortex ring pinches off at or close to the time that the piston stops moving, the occurrence of the TPM can be obscured by the low-pressure wave caused by the impulsive piston stop. This occurs for the $t_{a}^{*}=6$ velocity profile, as shown in Fig. 5, as well as for $t_{a}^{*}=8$. However, if we fit a line to the local maximum of the high-pressure region after the leading ring has fully formed, as indicated by the black line in Fig. 5, and extrapolate it backward in time to the nozzle exit plane at $X / D=0$, it is evident that this line coincides with a small region of high pressure at the nozzle exit that appears precisely at the time of vortex ring pinch-off, $t^{*}=6.6 \pm 0.1$.

Using this method to estimate the appearance of the dominant TPM for all velocity profiles examined in this study, Fig. 6 compares the time of TPM appearance to the calculated formation numbers based on the traditional circulation analysis. The estimate based on the TPM follows similar trends to the formation number as a function of piston acceleration distance. A longer piston acceleration results in both a higher formation number and a later TPM occurrence time. Additionally, at the largest acceleration distances tested, the occurrence of the TPM and the formation number both begin to plateau. For acceleration distances below saturation, the TPM occurs before pinch-off for most cases. This is consistent with the hypothesis that the formation of a high-pressure region behind the vortex ring causes pinch-off.

The present analysis of pressure in the shear layer is motivated by the fact that pinch-off depends on the entrainment of shear layer vorticity [5]. Nonetheless, a similar analysis can be conducted along the centerline, as in Lawson and Dawson [11]. Using the X-T technique to identify the appearance 
PRESSURE EVOLUTION IN THE SHEAR LAYER OF . .

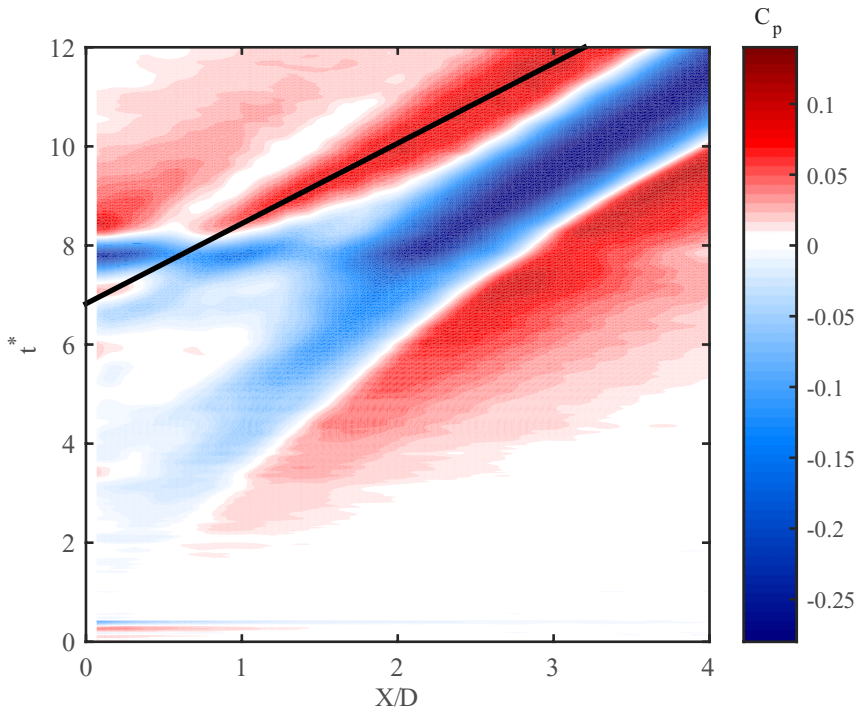

FIG. 5. Pressure evolution in the shear layer, $t_{a}^{*}=6$. Pressure coefficient $C_{p}=P / \rho u_{\max }^{2}$.

of the TPM at the nozzle exit plane results in a TPM occurrence time that is approximately 0.1 dimensionless time units before the TPM occurrence time in the shear layer. This discrepancy can potentially be much larger in general, as the centerline flow can deviate significantly from the shear layer in more complex flows [16].

\section{CONCLUSIONS}

Measurements of the evolution of pressure in the shear layer of developing vortex rings demonstrated a robust signature of vortex ring pinch-off, namely, a trailing pressure maximum

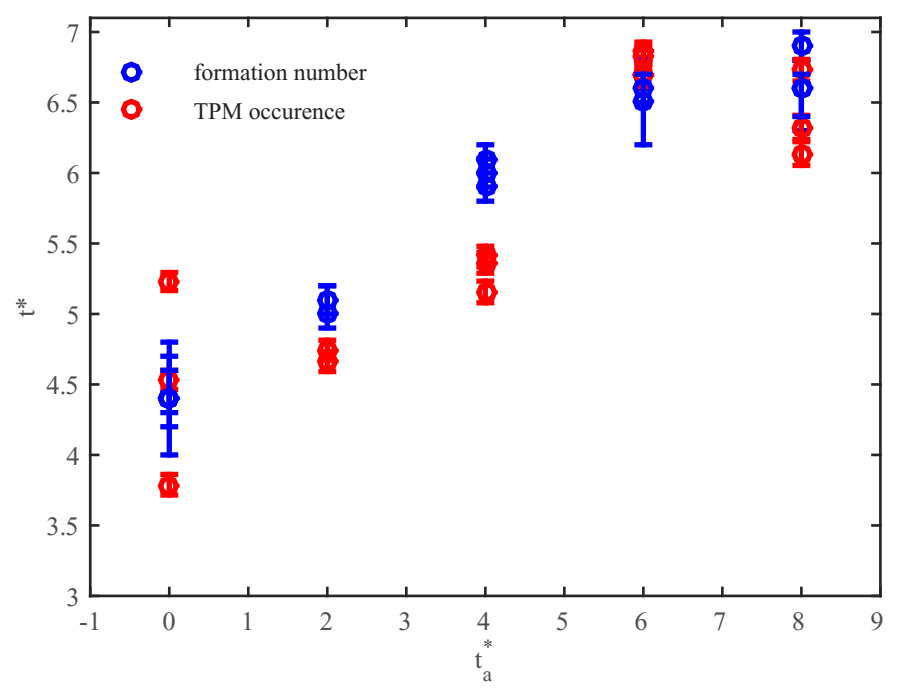

FIG. 6. Formation number (blue circles) and TPM occurrence time (red circles) as a function of acceleration distance. 


\section{KRISTY SCHLUETER-KUCK AND JOHN O. DABIRI}

that grows in magnitude to eventually become the high-pressure region directly behind the fully developed leading vortex ring. The appearance of a trailing pressure maximum is in general a necessary condition, but not a sufficient condition for determining if pinch-off has occurred, as it is possible for a TPM to appear transiently at the nozzle exit plane but eventually decay due to vortex merger, as seen in the constant velocity test case illustrated by Fig. 4. Physically, the presence of a robust TPM is consistent with pinch-off kinematics, as the adverse pressure gradient prevents the entrainment of shear layer fluid on the opposite side of the TPM from the leading vortex ring.

A method was developed to estimate the formation time when the growing TPM appears at the nozzle exit plane, namely, extrapolating the location of the high-pressure region behind the fully developed leading vortex ring backward in time to the nozzle exit plane based on the observed motion of the vortex ring. Both the TPM appearance estimated by this method and the formation number exhibited similar trends over the range of acceleration distances investigated.

\section{ACKNOWLEDGMENTS}

This work was supported by the Department of Defense (DoD) through the National Defense Science \& Engineering Graduate Fellowship (NDSEG) Program.

[1] T. Maxworthy, Turbulent vortex rings, J. Fluid Mech. 64, 227 (1974).

[2] K. Shariff and A. Leonard, Vortex rings, Annu. Rev. Fluid Mech. 24, 235 (1992).

[3] T. Lim and T. Nickels, Fluid Vortices (Kluwer Academic Publishers, Dordrecht, 1995), pp. 95-154.

[4] P. F. Linden and J. S. Turner, The formation of optimal vortex rings, and the efficiency of propulsion devices, J. Fluid Mech. 427, 61 (2001).

[5] M. Gharib, E. Rambod, and K. Shariff, A universal time scale for vortex ring formation, J. Fluid Mech. 360, 121 (1998).

[6] J. Dabiri and M. Gharib, Starting flow through nozzles with temporally variable exit diameter, J. Fluid Mech. 538, 111 (2005).

[7] K. Mohseni, H. Ran, and T. Colonius, Numerical experiments on vortex ring formation, J. Fluid Mech. 430, 267 (2001).

[8] P. Krueger, J. Dabiri, and M. Gharib, The formation number if vortex rings formed in uniform background co-flow, J. Fluid Mech. 556, 147 (2006).

[9] M. Shusser and M. Gharib, Energy and velocity of a forming vortex ring, Phys. Fluids 12, 618 (2000).

[10] L. Gao and S. Yu, Development of the trailing shear layer in a starting jet during pinch-off, J. Fluid Mech. 700, 382 (2012).

[11] J. M. Lawson and J. R. Dawson, The formation of turbulent vortex rings by synthetic jets, Phys. Fluids 25, 105113 (2013).

[12] J. Dabiri, S. Bose, B. J. Gemmell, S. P. Colin, and J. H. Costello, An algorithm to estimate unsteady and quasi-steady pressure fields from velocity field measurements, J. Exp. Biol. 217, 331 (2014).

[13] X. Liu and J. Katz, Instantaneous pressure and material acceleration measurements using a four exposure PIV system, Exp. Fluids 41, 227 (2006).

[14] B. W. van Oudheusden, PIV-based pressure measurement, Meas. Sci. Technol. 24, 032001 (2013).

[15] F. B. Kaplanski and Y. A. Rudi, A model for the formation of 'optimal' vortex rings taking into account viscosity, Phys. Fluids 17, 087101 (2005).

[16] J. Dabiri, S. P. Colin, and M. Gharib, Flow patterns generated by oblate medusan jellyfish: field measurements and laboratory analyses, J. Exp. Biol. 208, 1257 (2005). 